\title{
Improved Channel Estimation and SIR Measurement in WCDMA Downlink Systems
}

\author{
Ji-Woong Choi, Member, IEEE, Young-Shin Yoon, and Yong-Hwan Lee, Member, IEEE
}

\begin{abstract}
The rake receiver performance of a direct-sequence code-division multiple-access (DS-CDMA) system can significantly be degraded unless the channel condition is properly estimated. Since the channel condition is time-varying and location-dependent, it is desirable to employ an adaptive channel estimator (ACE) that can properly adjust the bandwidth of the channel estimation filter (CEF) according to the channel condition such as the maximum Doppler frequency. To this end, we propose low-complexity ACE and signal-to-interference power ratio (SIR) estimator for wide-band CDMA downlink systems with and without the use of transmit antenna diversity. The proposed schemes adjust the bandwidth of the CEF by exploiting the correlation characteristics of the received pilot signal. The analytic design is verified by computer simulation. Simulation results show that the proposed ACE and SIR estimator can significantly improve the link performance for a wide range of channel condition, particularly when the channel condition is poor.
\end{abstract}

Index Terms-Channel estimation, power control, signal-to-interference power ratio (SIR) measurement, wide-band code-division multiple access (WCDMA).

\section{INTRODUCTION}

$\mathbf{T}$ HE rake receiver in direct-sequence code-division multiple-access (DS-CDMA) systems requires the channel impulse response (CIR) for coherent demodulation and estimation of the signal-to-interference power ratio (SIR) [1]. To estimate the CIR, known pilot signals are usually transmitted being time-multiplexed or code-multiplexed with data signal [2], [3]. The third-generation (3G) wide-band CDMA (WCDMA) system adopts the use of a code-multiplexed method for common pilot channel and a time-multiplexed method for dedicated channels in the downlink [2]. To improve the accuracy of channel estimation, the received CIR needs to reduce the noise effect by employing a so-called channel estimation filter (CEF). It was reported that the characteristics of the CEF significantly affect the channel estimation performance and thus the receiver performance [4]. When a fixed CEF is employed, its cutoff frequency should be large enough to preserve the desired signal whose spectrum is spread to an allowable maximum Doppler frequency. However, if the

Manuscript received January 10, 2003; revised November 3, 2003. This work was presented in part at the IEEE International Symposium on Personal, Indoor, and Mobile Radio Communications, September 2002, and the IEEE Vehicular Technology Conference, May 2002. Review of this paper was coordinated by Dr. Z. (Daniel) Xu.

J.-W. Choi and Y.-H. Lee are with the School of Electrical Engineering and Computer Science and INMC, Seoul National University, Seoul 151-744, Korea (e-mail: jwch@ fruit.snu.ac.kr; ylee@ @nu.ac.kr).

Y.-S. Yoon is with SK Telecom, Seoul 100-768, Korea (e-mail: ysyoon@ sktelecom.com).

Digital Object Identifier 10.1109/TVT.2004.841525 cutoff frequency of the CEF is much larger than the maximum Doppler frequency of the experiencing channel, the CEF may produce an output with excessive noise, yielding significant performance degradation. This problem can be alleviated by adjusting the coefficient of the CEF in real time in response to the channel condition.

When the channel condition is known, Wiener filter is the optimum CEF in a linear minimum mean square error sense [5]. Since it requires a large computational complexity as well as exact information on the signal and noise, it may not be applicable in practice. As a result, simple CEFs, such as moving average (MA) finite impulse response (FIR) filter or one-pole infinite impulse response (IIR) filter, are usually employed in practice [6], [7]. They can provide relatively good channel estimation performance if they are appropriately designed according to the channel condition. Moreover, it can easily be designed by changing a single parameter, i.e., the tap size of MA FIR filter or the forgetting factor of one-pole IIR filter. Recently, there have been proposed several adaptive channel estimator (ACE) schemes that employ a simple CEF such as the MA FIR filter [8]-[10]. A simple ACE was proposed that chooses one of the two CEFs based on the estimated speed of channel variation [9]. The CEF can be selected among several CEFs according to the estimated speed zone [8], [10]. Since they consider only non-line-of-sight Rayleigh fading with the classic spectrum, the accuracy of speed estimation may not be acceptable under certain channel condition [8]-[10]. Moreover, since the CEFs are designed in a heuristic manner, their performance may not be improved significantly even though the maximum Doppler frequency is properly estimated.

To mitigate this problem, we consider design of an ACE in an analytic manner. To the best knowledge of the authors, few results have been reported on the analytic design of MA FIR CEF except some simulation results [11], [12]. In this paper, we first analytically design the optimum MA FIR CEF by minimizing the mean squared error (MSE) of the estimated CIR. Since the maximum Doppler frequency is the most critical factor in determining the bandwidth (i.e., the tap size) of the CEF, we estimate it using the correlation property of the received pilot symbol. To this end, we also analytically determine the optimum tap size of MA CEF as a function of the correlator output. Based on this analytic result, we design an ACE with low complexity.

The WCDMA system experiences the near-far problem due to the multiple-access interference (MAI) and fading effect, causing significant fluctuation in the received signal power [13]. These problems can be alleviated by adjusting the transmit power based on the estimated SIR of the received signal. Conventional schemes estimate the SIR of the received 


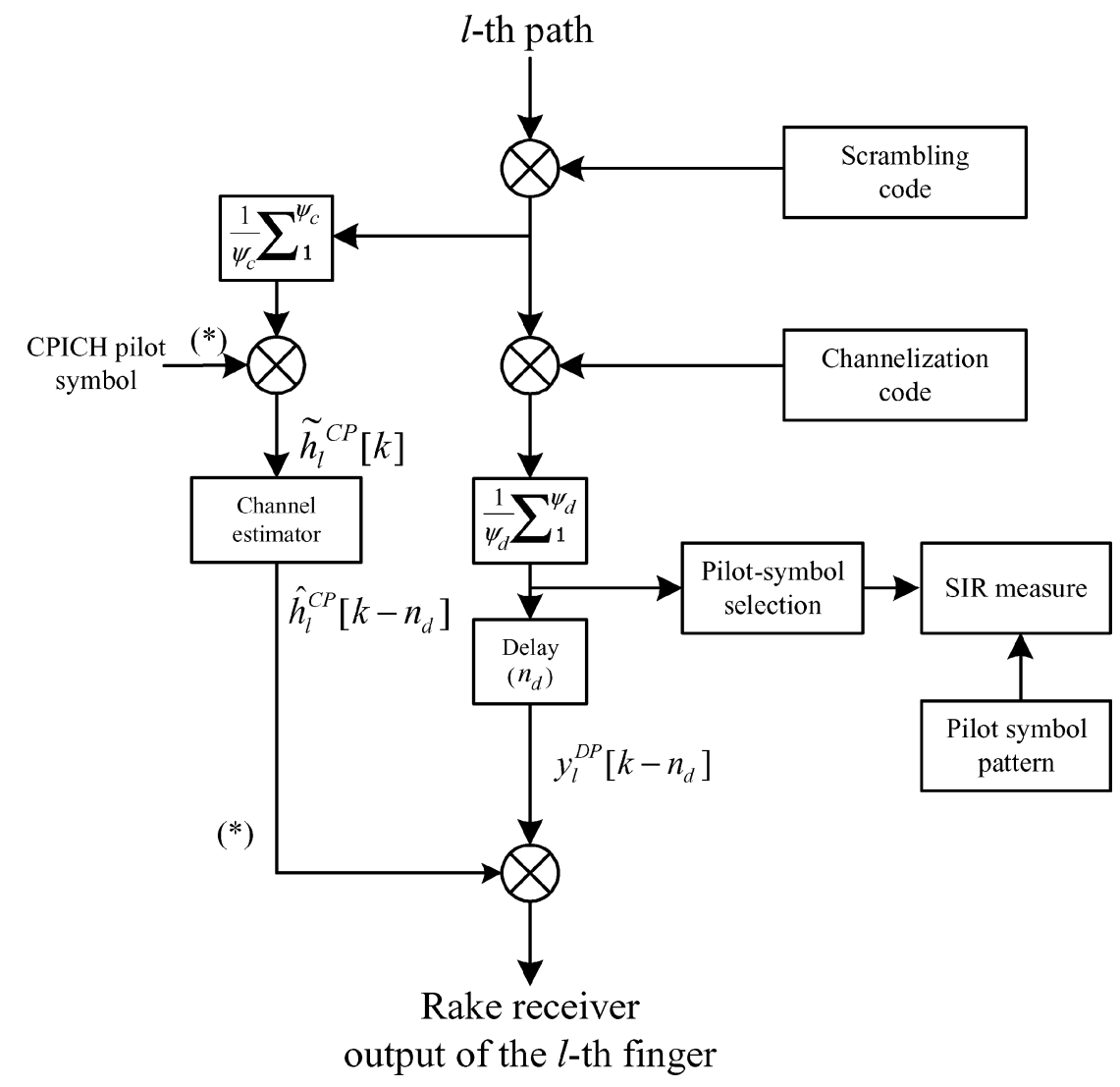

Fig. 1. The $l$ th branch of a rake receiver of WCDMA downlink system without transmit antenna diversity scheme.

signal by averaging the received pilot symbols over one slot time duration regardless of the channel condition [14]. Since the characteristics of the received signal and interference are affected by the fading condition, the accuracy of SIR measurement can be improved if the averaging interval of the received pilot symbol is adjusted in response to the channel condition [15]. In this paper, we also determine the averaging interval for SIR measurement in an analytic manner similar to the ACE.

Since it may not be practical to employ multiple antennas at the mobile handset, transmit antenna diversity (TAD) is usually employed at the base station in the WCDMA downlink system [2]. In this case, the receiver should estimate multiple CIRs in proportion to the number of transmit antennas. Since the total transmit power is split into multiple transmit antennas, however, the estimated CIR becomes less accurate, requiring an efficient channel estimation scheme. To investigate the effect of TAD on the channel estimation, we apply the proposed scheme to an open-loop TAD system [i.e., space time transmit diversity (STTD)] as well as single transmit antenna system.

Section II describes the transceiver structure in the WCDMA downlink. The proposed ACE and SIR estimator are described and their performance is evaluated without and with the use of TAD in Section III and IV, respectively. Conclusions are summarized in Section V.

\section{WCDMA DOWNLINK SYSTEM}

We consider two WCDMA downlink systems: single transmit antenna scheme and open-loop two-antenna TAD (or STTD) scheme [2]. Note that the proposed scheme can easily be applied to a closed-loop TAD scheme since it still employs the same common pilot channel (CPICH) and dedicated physical channel (DPCH) except different DPCH power for each transmit antenna. The proposed scheme can also be applied with a small modification to other DS-CDMA systems, including the IS-2000 system, that employ continuous common pilot signal and time-multiplexed user-dedicated pilot signal [3].

\section{A. WCDMA With Single Transmit Antenna}

The data and dedicated pilot symbol of each user are transmitted through a DPCH in a time-multiplexing manner. The DPCH symbols are spread using a user-allocated channelization code and the predetermined pilot bit of the $\mathrm{CPICH}$ is spread using a pilot channelization code. Each spread sequence is multiplied by the transmit gain and then aggregated before being scrambled. Finally, the aggregated signal is multiplied by a cell-specific scrambling code and then transmitted after pulse-shaping.

Assuming that there are $L$ independent propagation paths with different channel gain and time delay, the impulse response $h(t, \tau)$ of the channel at time $t$ can be represented as

$$
h(t, \tau)=\sum_{l=0}^{L-1} h_{l}(t) \delta\left(\tau-\tau_{l}\right)
$$

where $\delta(\tau)$ is Dirac delta function and $\tau_{l}$ denotes the delay of the $l$ th path signal. The impulse response $h_{l}(t)$ of the $l$ th path can be represented by

$$
h_{l}(t)=\alpha_{l}(t) e^{j \phi_{l}(t)}
$$


where $\alpha_{l}(t)$ and $\phi_{l}(t)$ are the amplitude and phase response of the $l$ th path at time $t$, respectively. We assume that the amplitude $\alpha_{l}(t)$ is Ricean distributed with Ricean factor $K_{l}$ and $\phi_{l}(t)$ is uniformly distributed over $[0,2 \pi)$ [16]. Denoting $P_{l}$ and $\theta_{l}$ by the power and arrival angle of the specular component and $\sigma_{\alpha, l}^{2}$ by the average power of the scattered component, $K_{l}$ is equal to $P_{l} / \sigma_{\alpha, l}^{2}$. Without loss of generality, we assume that the CIR has unit power

$$
E\left\{\sum_{l=0}^{L-1}\left|h_{l}(t)\right|^{2}\right\}=\sum_{l=0}^{L-1}\left(P_{l}+\sigma_{\alpha, l}^{2}\right)=1
$$

where $E\{x\}$ denotes the ensemble average of $x$.

The received signal $r(t)$ can be represented as

$$
r(t)=\sum_{l=0}^{L-1} h_{l}(t) x\left(t-\tau_{l}\right)+z(t)+\nu(t)
$$

where $x(t)$ is the signal transmitted from the desired cell with average received power spectral density $I_{o r}, z(t)$ denotes the received intercell interference from the adjacent cells with average power spectral density $I_{o r}$, and $\nu(t)$ is the background noise term. Using the central limit theorem, the intercell interference can be approximated as an additive white Gaussian noise (AWGN) term. Assuming that there are many users in service, we can ignore the background noise term.

Fig. 1 depicts the $l$ th branch of a rake receiver in the downlink. Denoting $y_{l}^{\mathrm{DP}}[k]$ by the received DPCH symbol despread at time $t=k T$ of the $l$ th branch, it can be obtained by multiplying the descrambled signal with the DPCH channelization code and then accumulating for an amount of $\psi_{d}$ samples. The CPICH signal is accumulated for an amount of $\psi_{c}(=256)$ samples and then multiplied with the predetermined pilot symbol to obtain the despread CPICH symbol $\tilde{h}_{l}^{\mathrm{CP}}[k]$. Assume that the $\mathrm{CPICH}$ transmit power is $\beta_{c}^{2}$ times the total transmit power at the base station, i.e., $\beta_{c}^{2}$ is equal to $E_{c} / I_{o r}$ of the $\mathrm{CPICH}$, where $E_{c}$ is the chip energy. Then, the despread CPICH symbol can be represented as

$$
\tilde{h}_{l}^{\mathrm{CP}}[k]=\beta_{c} \sqrt{I_{o r}} h_{l}[k]+z_{l}^{\mathrm{CP}}[k]
$$

where $z_{l}^{\mathrm{CP}}[k]$ is the $l$ th path interference of the despread $\mathrm{CPICH}$ symbol approximated as AWGN with variance $\left(I_{o c}+v_{l} I_{o r}\right) \psi_{c}^{-1}$. Here, $v_{l}$ is the orthogonality factor corresponding to the $l$ th path, i.e., the ratio of the interference to total power from the desired cell. For example, $v_{l}$ is equal to zero under flat fading condition and approaches one as the number of multipaths increases.

The desired DPCH symbol can be obtained by

$$
\hat{s}^{\mathrm{DP}}\left[k-n_{d}\right]=\sum_{l=0}^{L-1} \hat{h}_{l}^{C P *}\left[k-n_{d}\right] y_{l}^{\mathrm{DP}}\left[k-n_{d}\right]
$$

where $n_{d}$ denotes the delay due to channel estimation, $\hat{h}_{l}^{\mathrm{CP}}[k]$ is the output of the channel estimator obtained from the CPICH, and the superscript $*$ denotes the complex conjugate. When a $\left(2 N_{l}+1\right)$-tap FIR filter is employed as the CEF, $\hat{h}_{l}^{\mathrm{CP}}[k]$ can be represented as

$$
\hat{h}_{l}^{\mathrm{CP}}[k]=\sum_{i=-N_{l}}^{N_{l}} c_{l, i} \tilde{h}_{l}^{\mathrm{CP}}[k+i] .
$$

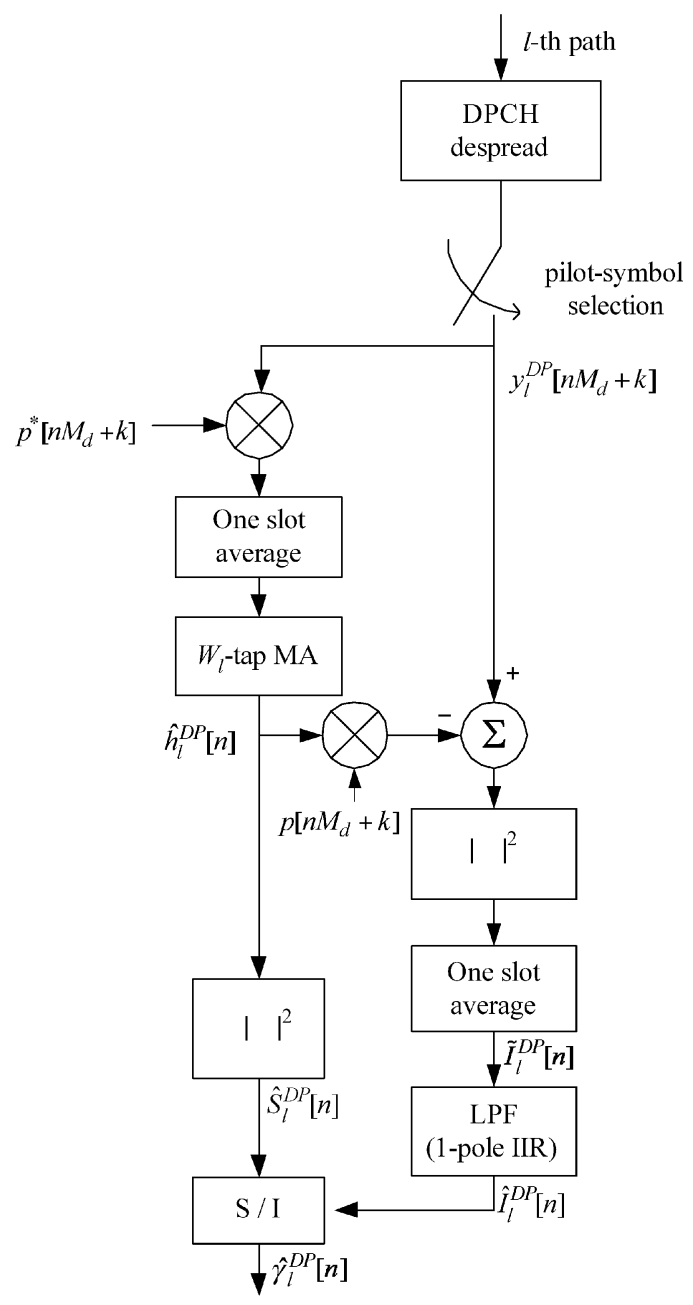

Fig. 2. Structure of SIR estimator of the $l$ th branch.

It was shown that the use of general FIR filters can provide a marginal gain over the use of MA FIR filters [17]. Both the MA and general FIR filters require the same amount of memory and addition operation, but the MA FIR CEF requires only a single multiplication for normalization. Thus, we consider the use of MA FIR CEFs with $c_{l, i}=\left(2 N_{l}+1\right)^{-1}$ due to its simplicity of implementation, while providing relatively good performance.

Fig. 2 depicts a block diagram of the SIR estimation scheme for the $l$ th branch in a rake receiver, where the pilot symbol in the DPCH is used for SIR measurement. Note that the pilot symbol in the CPICH cannot be used for SIR measurement since all the users use the same CPICH. Assuming that the DPCH transmit power is $\beta_{d}^{2}$ times the total transmit power of the base station, $\beta_{d}^{2}$ is equal to $E_{c} / I_{o r}$ of the DPCH. During the transmission of the dedicated pilot symbol in the $n$th slot, the $l$ th path despread pilot signal in the DPCH can be represented as

$$
\begin{aligned}
& y_{l}^{\mathrm{DP}}\left[n M_{d}+k\right]=\beta_{d} \sqrt{I_{o r}} h_{l}\left[n M_{d}+k\right] p\left[n M_{d}+k\right] \\
& \quad+z_{l}^{\mathrm{DP}}\left[n M_{d}+k\right], \quad M_{d}-M_{p} \leq k \leq M_{d}-1
\end{aligned}
$$

where $M_{d}$ is the number of total DPCH symbols per slot, i.e., $M_{d}=2560 / \psi_{d}, M_{p}$ is the number of dedicated pilot symbols per slot, $h_{l}\left[n M_{d}+k\right]$ is the $l$ th path normalized CIR of the $k$ th symbol in the $n$th slot, $p\left[n M_{d}+k\right]$ is the dedicated pilot symbol with unit power (i.e., $\left|p\left[n M_{d}+k\right]\right|=1$ ), and $z_{l}^{\mathrm{DP}}\left[n M_{d}+\right.$ 
$k$ ] denotes the interference term approximated as AWGN with variance $\left(I_{o c}+v_{l} I_{o r}\right) \psi_{d}^{-1}$.

The estimated signal power $\hat{S}_{l}^{\mathrm{DP}}[n]$ of the $n$th slot in the $l$ th path can be obtained by [14]

$$
\hat{S}_{l}^{\mathrm{DP}}[n]=\left|\hat{h}_{l}^{\mathrm{DP}}[n]\right|^{2}
$$

where $\hat{h}_{l}^{\mathrm{DP}}[n]$ is the CIR corresponding to the $n$th slot of the $\mathrm{DPCH}$

$$
\begin{aligned}
& \hat{h}_{l}^{\mathrm{DP}}[n]= \frac{1}{W_{l}} \sum_{i=0}^{W_{l}-1} \tilde{h}_{l}^{\mathrm{DP}}[n-i] \\
&=\frac{1}{W_{l}} \sum_{i=0}^{W_{l}-1}\left(\frac{1}{M_{P}} \sum_{k=M_{d}-M_{p}}^{M_{d}-1} y_{l}^{\mathrm{DP}}\left[(n-i) M_{d}+k\right]\right. \\
&\left.\cdot p^{*}\left[(n-i) M_{d}+k\right]\right) .
\end{aligned}
$$

Here, $W_{l}$ is the number of slots for averaging. In a conventional nonadaptive scheme, $W_{l}$ is usually fixed to one [14].

Instantaneous interference of the $n$th slot of the DPCH can be obtained by

$$
\begin{array}{r}
\tilde{I}_{l}^{\mathrm{DP}}[n]=\frac{1}{M_{P}} \sum_{k=M_{d}-M_{p}}^{M_{d}-1} \mid y_{l}^{\mathrm{DP}}\left[n M_{d}+k\right] \\
-\left.\hat{h}_{l}^{\mathrm{DP}}[n] p\left[n M_{d}+k\right]\right|^{2} .
\end{array}
$$

The inteference power can be estimated as [14]

$$
\hat{I}_{l}^{\mathrm{DP}}[n]=\alpha_{\operatorname{sir}} \hat{I}_{l}^{\mathrm{DP}}[n-1]+\left(1-\alpha_{\text {sir }}\right) \tilde{I}_{l}^{\mathrm{DP}}[n]
$$

where $\alpha_{\text {sir }}$ is the forgetting factor for interference measurement. The SIR of the $n$th slot of the $l$ th path is estimated by

$$
\hat{\gamma}_{l}^{\mathrm{DP}}[n]=\frac{\hat{S}_{l}^{\mathrm{DP}}[n]}{\hat{I}_{l}^{\mathrm{DP}}[n]} .
$$

Finally, the overall SIR from all the resolvable paths is estimated as

$$
\hat{\gamma}_{t}^{\mathrm{DP}}[n]=\sum_{l=0}^{L-1} \hat{\gamma}_{l}^{\mathrm{DP}}[n] .
$$

The power-control command is determined by comparing $\hat{\gamma}_{t}^{\mathrm{DP}}[n]$ with a predetermined threshold.

\section{B. WCDMA With Open-Loop Two-Transmit Antennas}

We consider a two-antenna STTD system, where the DPCH data $x_{i}(t)$ are transmitted through antenna- $i, i=1,2$, as [18]

$$
\begin{aligned}
& x_{1}(t)= \begin{cases}s_{1}[k], & 2 k T \leq t<(2 k+1) T \\
s_{2}[k], & (2 k+1) T \leq t<(2 k+2) T\end{cases} \\
& x_{2}(t)= \begin{cases}-s_{2}^{*}[k], & 2 k T \leq t<(2 k+1) T \\
s_{1}^{*}[k], & (2 k+1) T \leq t<(2 k+2) T .\end{cases}
\end{aligned}
$$

The orthogonal pilot symbol sets $[1,1]$ and $[1,-1]$ (or $[-1$, 1]) are, respectively, transmitted through the $\mathrm{CPICH}$ in parallel with the DPCH at antenna-1 and -2 over a two-symbol time interval. Other procedures are done similarly as in the single transmit antenna scheme. It can be assumed that the channel between each transmit antenna and receive antenna experiences independent fading, provided that transmit antennas are spaced sufficiently apart [18].

Let $y_{1, l}^{\mathrm{DP}}[k]$ and $y_{2, l}^{\mathrm{DP}}[k]$ be the received DPCH symbol despread at $t=2 k T$ and $t=(2 k+1) T$ of the $l$ th path, respectively

$$
\begin{aligned}
y_{1, l}^{\mathrm{DP}}[k]= & \beta_{d, 1} \sqrt{I_{o r}} h_{l, 1}[k] s_{1}[k]-\beta_{d, 2} \sqrt{I_{o r}} h_{l, 2}[k] s_{2}^{*}[k] \\
& +z_{1, l}^{\mathrm{DP}}[k] \\
y_{2, l}^{\mathrm{DP}}[k]= & \beta_{d, 1} \sqrt{I_{o r}} h_{l, 1}[k] s_{2}[k]+\beta_{d, 2} \sqrt{I_{o r}} h_{l, 2}[k] s_{1}^{*}[k] \\
& +z_{2, l}^{\mathrm{DP}}[k]
\end{aligned}
$$

where $h_{l, i}[k]$ is the normalized CIR of the $l$ th path between the receiver antenna and transmit antenna $i$ and $z_{1, l}^{\mathrm{DP}}[k]$ and $z_{2, l}^{\mathrm{DP}}[k]$ are the interference in the $l$ th path at time $t=2 k T$ and $(2 k+1) T$, respectively. Here, we assume that the CIR is unchanged during two-symbol time interval. In the STTD scheme, each antenna has the same transmit gain, i.e., $\beta_{d, 1}=\beta_{d, 2}(=$ $\left.\beta_{d} / \sqrt{2}\right)$, while $\beta_{d, 1} \neq \beta_{d, 2}$ in a closed-loop TAD scheme. The despread CPICH symbols $y_{1, l}^{\mathrm{CP}}[k]$ and $y_{2, l}^{\mathrm{CP}}[k]$ of the $l$ th path at $t=2 k T$ and $t=(2 k+1) T$ are, respectively, given by

$$
\begin{aligned}
& y_{1, l}^{\mathrm{CP}}[k]=\beta_{c, 1} \sqrt{I_{o r}} h_{l, 1}[k]+\beta_{c, 2} \sqrt{I_{o r}} h_{l, 2}[k]+z_{1, l}^{\mathrm{CP}}[k] \\
& y_{2, l}^{\mathrm{CP}}[k]=\beta_{c, 1} \sqrt{I_{o r}} h_{l, 1}[k]-\beta_{c, 2} \sqrt{I_{o r}} h_{l, 2}[k]+z_{2, l}^{\mathrm{CP}}[k] .
\end{aligned}
$$

Using instantaneous CIR estimate $\tilde{h}_{l, i}^{\mathrm{CP}}[k]$ of antenna- $i$

$$
\begin{aligned}
& \tilde{h}_{l, 1}^{\mathrm{CP}}[k]=\frac{1}{2}\left(y_{1, l}^{\mathrm{CP}}[k]+y_{2, l}^{\mathrm{CP}}[k]\right) \\
& \tilde{h}_{l, 2}^{\mathrm{CP}}[k]=\frac{1}{2}\left(y_{1, l}^{\mathrm{CP}}[k]-y_{2, l}^{\mathrm{CP}}[k]\right)
\end{aligned}
$$

the corresponding CIR can be estimated as

$$
\hat{h}_{l, i}^{\mathrm{CP}}[k]=\frac{1}{\left(2 N_{l}^{S T}+1\right)} \sum_{j=-N_{l}^{S T}}^{N_{l}^{S T}} \tilde{h}_{l, i}^{\mathrm{CP}}[k+j] .
$$

The desired DPCH data symbol can be obtained by

$$
\begin{array}{r}
\hat{s}_{1}\left[k-n_{d}\right]=\sum_{l=0}^{L-1}\left(\hat{h}_{l, 1}^{C P *}\left[k-n_{d}\right] y_{1, l}^{\mathrm{DP}}\left[k-n_{d}\right]\right. \\
\left.+\hat{h}_{l, 2}^{\mathrm{CP}}\left[k-n_{d}\right] y_{2, l}^{D *}\left[k-n_{d}\right]\right) \\
\hat{s}_{2}\left[k-n_{d}\right]=\sum_{l=0}^{L-1}\left(-\hat{h}_{l, 2}^{\mathrm{CP}}\left[k-n_{d}\right] y_{1, l}^{D *}\left[k-n_{d}\right]\right. \\
\left.+\hat{h}_{l, 1}^{C P *}\left[k-n_{d}\right] y_{2, l}^{\mathrm{DP}}\left[k-n_{d}\right]\right) .
\end{array}
$$

The SIR of the DPCH symbol can be estimated using the estimated DPCH CIR of each antenna as in the single transmit 


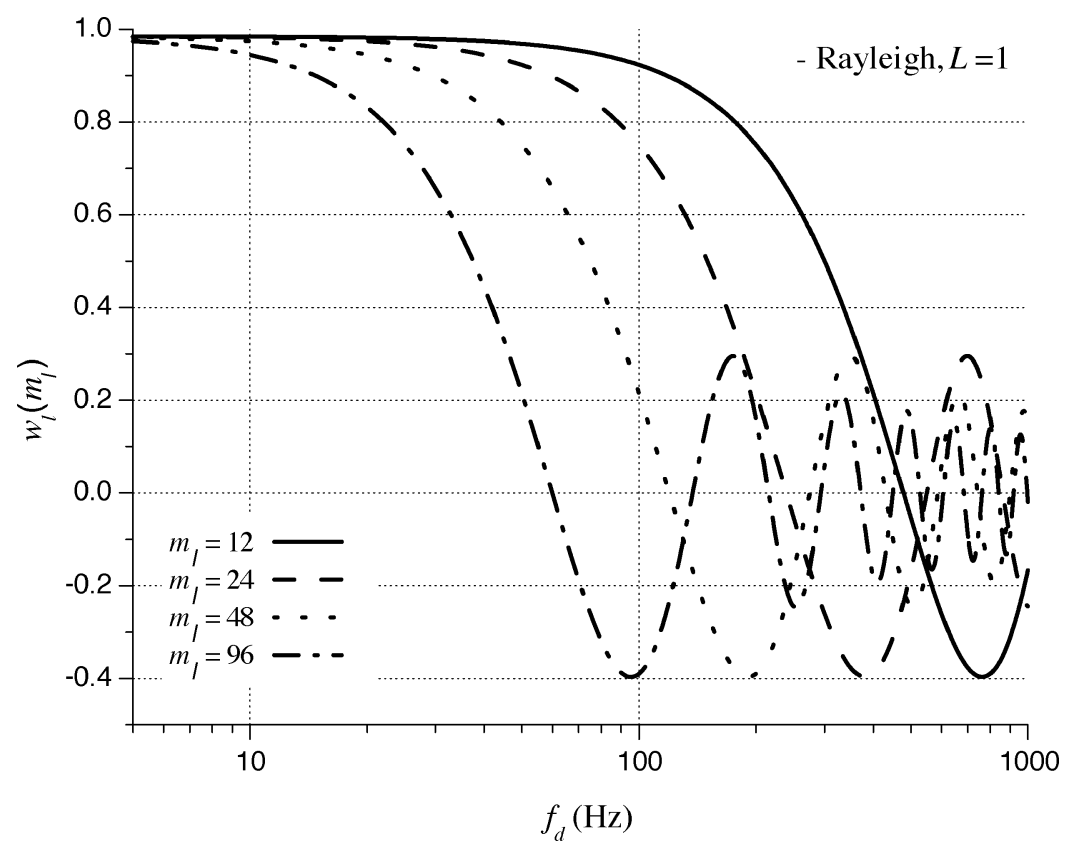

Fig. 3. The output of the correlator bank.

antenna scheme. The signal power $\hat{S}_{l}^{\mathrm{DP}}[n]$ of the $n$th slot of the $l$ th path can be estimated as

$$
\hat{S}_{l}^{\mathrm{DP}}[n]=\left|\hat{h}_{l, 1}^{\mathrm{DP}}[n]\right|^{2}+\left|\hat{h}_{l, 2}^{\mathrm{DP}}[n]\right|^{2}
$$

The interference power and overall SIR can be obtained similarly as in the single transmit antenna scheme.

\section{Channel Estimation And SIR Measurement For Single TRANSMIT ANTENNA}

\section{A. Proposed ACE Scheme}

We optimally determine the tap size $\hat{M}_{l}\left(=2 \hat{N}_{l}+1\right)$ of the MA FIR CEF that minimizes the MSE of the channel estimate defined as

$$
\varepsilon_{l}\left[N_{l}\right]=E\left\{\left|\hat{h}_{l}^{\mathrm{CP}}[k]-h_{l}^{\mathrm{CP}}[k]\right|^{2}\right\} .
$$

It can easily be shown that

$$
\begin{aligned}
\varepsilon_{l}\left[N_{l}\right]=\beta_{c}^{2} I_{o r} & \left(\frac{1}{\left(2 N_{l}+1\right)^{2}} \sum_{i=-N_{l}}^{N_{l}} \sum_{j=-N_{l}}^{N_{l}} R_{h, l}[i-j]\right. \\
& \left.-\frac{2}{2 N_{l}+1} \sum_{i=-N_{l}}^{N_{l}} R_{h, l}[i]+R_{h, l}[0]\right) \\
& +\frac{I_{o c}+v_{l} I_{o r}}{\left(2 N_{l}+1\right) \psi_{c}}
\end{aligned}
$$

where $R_{h, l}[i]$ is the real part of the autocorrelation of the $l$ th sampled CIR defined as

$$
R_{h, l}[i]=E\left\{\operatorname{Re}\left(h_{l}^{*}[k] h_{l}[k+i]\right)\right\} .
$$

The optimum tap size $\hat{M}_{l}$ minimizing the MSE can be determined as (refer to Appendix I)

$$
\hat{M}_{l}=\left(\frac{v_{l}+\frac{I_{o c}}{I_{o r}}}{\left(\pi f_{d} T_{c p}\right)^{4} \psi_{c} \beta_{c}^{2} S_{l}} \cdot \frac{1+K_{l}}{\frac{K_{l} \cos ^{4} \theta_{l}}{9}+\frac{1}{\chi_{l}}}\right)^{\frac{1}{5}}
$$

where $f_{d}$ is the maximum Doppler frequency, $S_{l}$ is the average power ratio of the $l$ th path signal to total signal, $T_{c p}$ is the time duration of the CPICH symbol, and $\chi_{l}$ is equal to 24 and 45 in the case of classic and flat spectrum, respectively. For a given $f_{d}$, the optimum tap size $\hat{M}_{l}$ decreases as $\beta_{c}, S_{l}, v_{l}^{-1}, I_{o r} / I_{o c}$, and/or $K_{l}$ increase, and vice versa. However, it can be seen that $f_{d}$ is most influential on $\hat{M}_{l}$ among these parameters. Thus, if $f_{d}$ is correctly estimated, the tap size can be determined without significant performance degradation even when other channel condition parameters are not exactly known.

The maximum Doppler frequency $f_{d}$ can be estimated by exploiting the correlation function of the received pilot signal over $J$ symbols

$$
w_{l}\left(m_{l}\right)=\frac{\sum_{k=0}^{J-1} \operatorname{Re}\left\{\bar{h}_{l}^{C P *}[k] \bar{h}_{l}^{\mathrm{CP}}\left[k+m_{l}\right]\right\}}{\sum_{k=0}^{J-1}\left|\bar{h}_{l}^{\mathrm{CP}}[k]\right|^{2}}
$$

where $\bar{h}_{l}^{\mathrm{CP}}[k]$ is the prefiltered output of $\tilde{h}_{l}^{\mathrm{CP}}[k]$ to suppress the noise components larger than the allowable maximum Doppler frequency. For simplicity of design, we consider the use of an $N_{a}$-tap MA FIR filter as the prefilter. Fig. 3 depicts the values of $w_{l}\left(m_{l}\right)$ for four different values of $m_{l}$ as a function of $f_{d}$ in a single-path Rayleigh fading channel when $\beta_{c}^{2}=-13 \mathrm{~dB}$, $I_{o r} / I_{o c}=0 \mathrm{~dB}, N_{a}=5$. It can be seen that, for a given $m_{l}, w_{l}\left(m_{l}\right)$ monotonically decreases as $f_{d}$ increases until it reaches the minimum value and that the larger $m_{l}$, the faster $w_{l}\left(m_{l}\right)$ decreases. Comparing the correlation output with a 


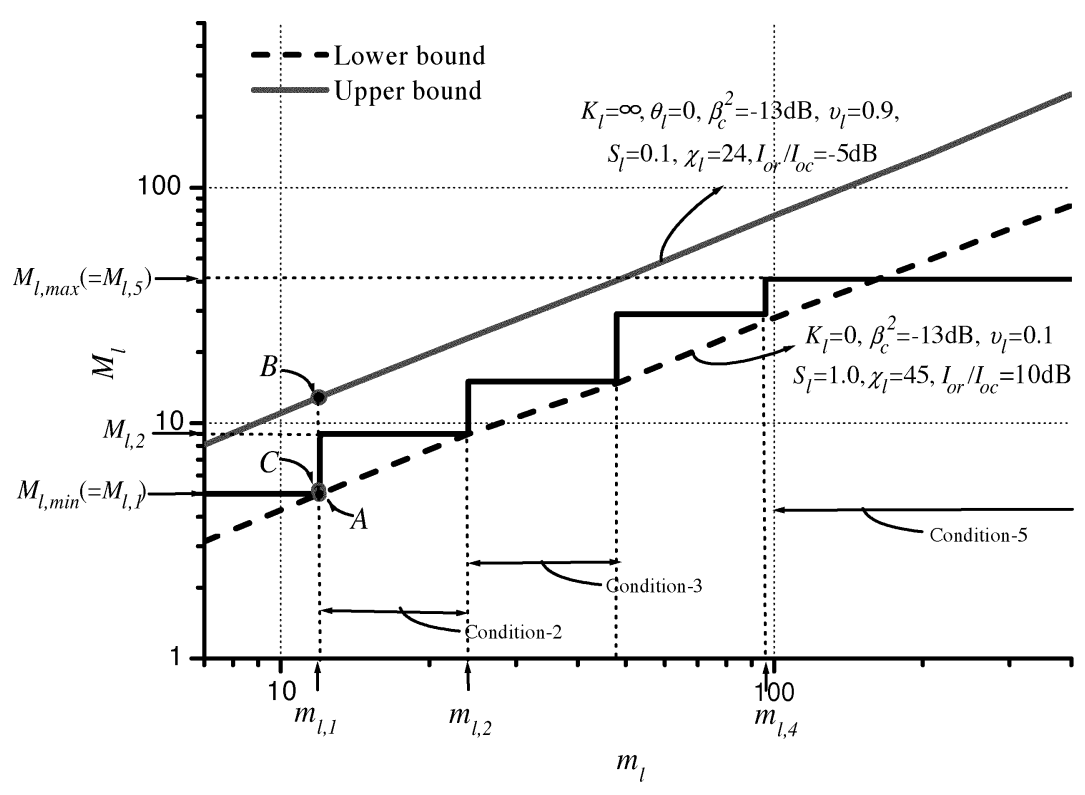

Fig. 4. The tap size of the MA FIR CEF.

given threshold $\eta$ for all integer values of $m_{l}$, we can find $\hat{m}_{l}$ satisfying $w_{l}\left(\hat{m}_{l}\right)=\eta$. For example, $\hat{m}_{l}=12,24,48$ and 96 with $\eta=0.3$ when $f_{d}=370,185,92$, and $46 \mathrm{~Hz}$, respectively.

The optimum tap size can be represented as a function of $\hat{m}_{l}$ (refer to Appendix II)

$$
\hat{M}_{l}=\left(\frac{16 \hat{m}_{l}^{4}\left(v_{l}+\frac{I_{o c}}{I_{o r}}\right)}{\psi_{c} \beta_{c}^{2} S_{l} \hat{f}_{l}^{4}} \cdot \frac{1+K_{l}}{\frac{K_{l} \cos ^{4} \theta_{l}}{9}+\frac{1}{\chi_{l}}}\right)^{\frac{1}{5}}
$$

where $\hat{f}_{l}$ is the normalized maximum Doppler frequency. Note that $\hat{f}_{l}$ is not a function of $f_{d}$ but a function of other channel condition parameters. The optimum tap size of MA FIR CEF needs to be determined considering other channel condition parameters as well as $\hat{m}_{l}$. As an example, Fig. 4 depicts the upper and lower bound of the tap size as a function of $\hat{m}_{l}$ in a typical channel condition, where $0 \leq K_{l}<\infty,-90^{\circ} \leq \theta_{l} \leq 90^{\circ}$, $\beta_{c}^{2}=-13 \mathrm{~dB},-5 \mathrm{~dB} \leq \bar{I}_{\text {or }} / \leq 10 \mathrm{~dB}, 0.1 \leq S_{l} \leq 1.0$, $0.1 \leq v_{l} \leq 0.9$, and $24 \leq \chi_{l} \leq 45$. It can be seen that the optimum tap size is not much affected by the channel parameters other than $f_{d}$. In practice, the tap size can be determined as an integer number between the upper and lower bound so that the ACE can provide near optimum performance by only estimating the speed of channel, i.e., obtaining $\hat{m}_{l}$ satisfying $w_{l}\left(\hat{m}_{l}\right)=\eta$, without consideration of other channel parameters.

However, it may not be practical to employ as many as correlators for all integer numbers of $m_{l}$ to find $\hat{m}_{l}$. Moreover, $\hat{m}_{l}$ can be a noninteger value. Thus, rather than accurately estimating the maximum Doppler frequency, we classify the channel condition into a small number of cases using a staircase approximation as shown in Fig. 4 , where $m_{l, i}$ is $\hat{m}_{l}$ specified in the staircase. Since $w_{l}\left(m_{i}\right)$ decreases faster than $w_{l}\left(m_{j}\right)$ for $m_{i}>m_{j}$, the channel condition can be classified by comparing $w_{l}\left(m_{l}\right)$ with $\eta$. If the $j$ th correlator output of the $l$ th branch becomes less than $\eta$ for the first time, i.e.,

$$
w_{l}\left(m_{l, j}\right)<\eta \text { and } w_{l}\left(m_{l, j-1}\right) \geq \eta, \quad j=1,2, \ldots, G_{l}
$$

we infer that the channel condition belongs to case- $j$. In this case, the tap size of the corresponding MA FIR CEF is set to $M_{l, j}, 1 \leq j \leq G_{l}$. Note that the tap size $M_{l, G_{l}+1}$ corresponds to the case when no correlator output is less than the threshold. This condition occurs when the channel response varies slowly.

\section{B. Proposed SIR Measurement Scheme}

Transmit power control requires accurate SIR measurement. Channel estimation of the DPCH required for SIR measurement can be done similar to that of the CPICH except the consideration of delay in channel estimation. The receiver performance is not affected by the delay due to channel estimation by adding the same amount of delay to the data path. However, the transmit power control should properly reflect the current channel condition. Thus, we cannot use the delayed output of the DPCH channel estimator for SIR measurement as (10). It can be possible to obtain a DPCH channel estimate without delay by modifying the calculation of the MSE using only the current and previous symbols. Similar to the derivation of (27), the optimum number of DPCH symbols can easily be obtained by

$$
\hat{M}_{l}^{\prime}=\left(\frac{\hat{m}_{l}^{2}\left(v_{l}+\frac{I_{o c}}{I_{o r}}\right)}{\psi_{d} \beta_{d}^{2} S_{l} \hat{f}_{l}^{2}} \cdot \frac{1+K_{l}}{\frac{K_{l} \cos ^{2} \theta_{l}}{9}+\frac{1}{\chi_{l}}}\right)^{\frac{1}{3}} .
$$

Since the MA CEF for the SIR measurement is working for each slot as (10), the optimum number of slots $\hat{W}_{l}$ can be determined by

$$
\hat{W}_{l}=\left\lfloor\frac{\hat{M}_{l}^{\prime}}{M_{d}}\right\rfloor
$$

where $\lfloor x\rfloor$ denotes an integer less than or equal to $x$.

\section{Structure of the Proposed Scheme}

Fig. 5 depicts the proposed ACE and SIR estimator for the $l$ th branch of the rake receiver. The proposed scheme consists 


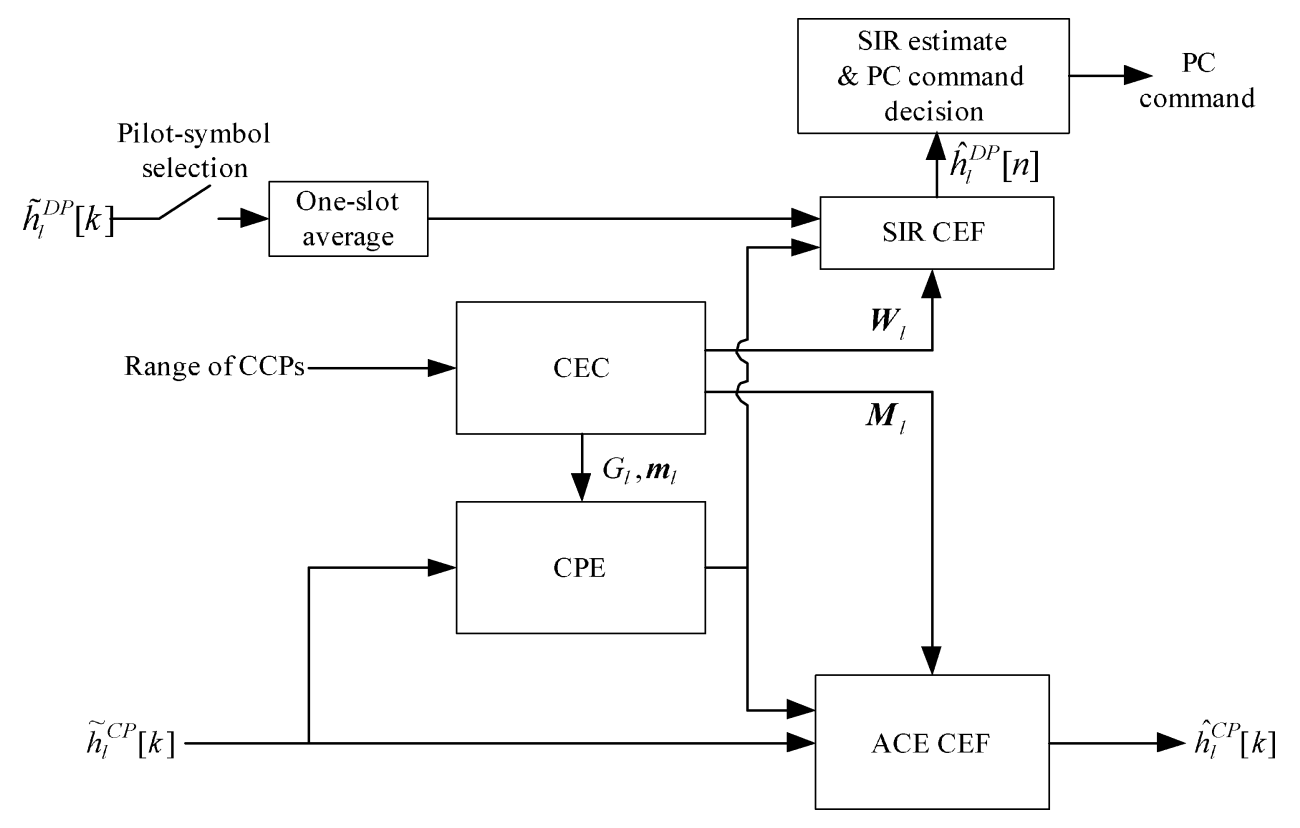

Fig. 5. Structure of the proposed ACE and SIR estimator.

of the channel parameter estimator (CPE), channel estimation controller (CEC), and two CEFs: one CEF for the ACE and the other CEF for the SIR estimation. The CPE determines the channel condition by (28) to control the tap size of the MA FIR CEF. When the mobile station is initialized, the values of $G_{l}$, $m_{l, i}, M_{l, i}$, and $W_{l, i}, i=1,2, \ldots, G_{l}$, are determined using a staircase approximation in the CEC by considering the range of the channel condition parameters (CCPs). The CEC considers the minimum and maximum values of the CCPs, i.e., $K_{l}, \theta_{l}, S_{l}$, $\beta_{c}$, and $\chi_{l}$, and other parameters such as $\eta, N_{a}, M_{l, \min }$, and $M_{l, \max }$. From these parameters, the upper and lower bound of the tap size can be determined using (27) as in Fig. 4. Based on the minimum and maximum value of the CCPs, a set of the correlator's interval and the tap size of the MA FIR CEF can be determined in a sequential manner. This process is described in Appendix III. Under the CCPs shown in Fig. 3, we can obtain $G_{l}=4, m_{l}=\{12,24,48,96\}, M_{l}=\{5,9,15,29,41\}$, and $W_{l}=\{1,1,1,2,3\}$. The tap size of the MA CEF is updated once every $J$ symbols as (26). The channel condition can be estimated with an acceptable accuracy by correlating symbols less than a few thousands. This takes time less than a few hundred milliseconds, enabling the use of the proposed scheme even when the channel environment varies fast.

It is possible to use one-pole IIR filters as the CEF for further reduction of the implementation complexity without noticeable performance degradation [17]. The frequency response of an MA FIR filter can be approximated by that of a one-pole IIR filter as [8], [19]

$$
\hat{h}_{l}[k]=\tilde{h}_{l}[k]+\mu_{l}\left(\hat{h}_{l}[k-1]-\tilde{h}_{l}[k]\right)
$$

where $\mu_{l}=1-\hat{N}_{l}^{-1}$. Thus, the results obtained with the use of MA FIR CEFs can directly be applied to the use of one-pole IIR CEFs.
TABLE I

\begin{tabular}{c|c}
\multicolumn{2}{c}{ Simulation Condition } \\
\hline Parameters & Values \\
\hline Data rate & $12.2,144 \mathrm{Kbps}$ \\
Chip rate & $3.84 \mathrm{Mcps}$ \\
$\psi_{d}$ & $128(12.2 \mathrm{Kbps}), 16(144 \mathrm{Kbps})$ \\
$\mathrm{CPICH} E_{c} / I_{o r}\left(\beta_{c}^{2}\right)$ & $-13 \mathrm{~dB}$ \\
$\mathrm{PCCPCH} E_{c} / I_{o r}$ & $-12 \mathrm{~dB}$ \\
$\mathrm{SCH} E_{c} / I_{o r}$ & $-12 \mathrm{~dB}$ \\
$\mathrm{PICH} E_{c} / I_{o r}$ & $-15 \mathrm{~dB}$ \\
Channel & $5 \mathrm{~Hz}-750 \mathrm{~Hz}$ \\
Doppler frequency $\left(f_{d}\right)$ & Step size $=1 \mathrm{~dB}$, control rate $=800 \mathrm{~Hz}$ \\
Power control
\end{tabular}

\section{Performance Evaluation}

The performance of the proposed ACE and SIR estimator is evaluated in terms of the bit error rate (BER) performance under various channel conditions. The simulation condition is summarized in Table I. We consider the Case-1 and Case-3 3GPP multipath channel models as a typical flat-like and frequency selective fading channel, respectively [20]. Rayleigh fading channel is generated using the Joint Technical Committee model [21], where the line-of-sight component is added in a form of sinusoidal wave [22]. Fig. 6 depicts the required DPCH $E_{c} / I_{o r}$ for a BER of $10^{-3}$ in Case- 3 channel when $I_{o r} / I_{o c}=-3 \mathrm{~dB}$ (i.e., the mobile is close to the cell boundary) and $9 \mathrm{~dB}$ (i.e., the mobile is near the base station). We assume that the fixed channel estimator (FCE) and fixed power controller (FPC) use a CEF with one slot averaging interval, that can accommodate the maximum Doppler frequency of up to $750 \mathrm{~Hz}$. For reference, the receiver performance is also evaluated under the ideal estimation condition, where the CIR of the CPICH and DPCH is perfectly known.

It can be seen that, as $f_{d}$ decreases, the performance gain of the proposed ACE over the FCE increases since the FCE is nearly optimum for high $f_{d}$. As $I_{o r} / I_{o c}$ decreases, the SIR of the despread CPICH pilot symbol decreases, increasing the performance differentiation between the use of the ACE and FCE. 


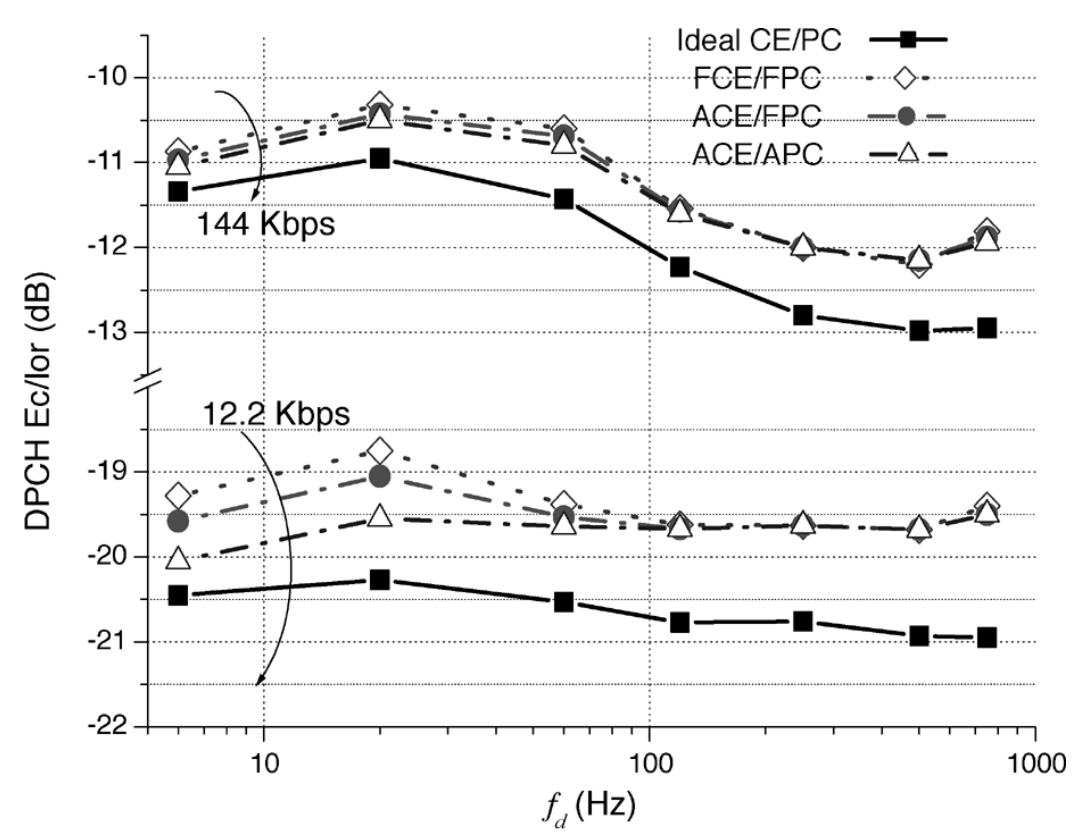

(a)

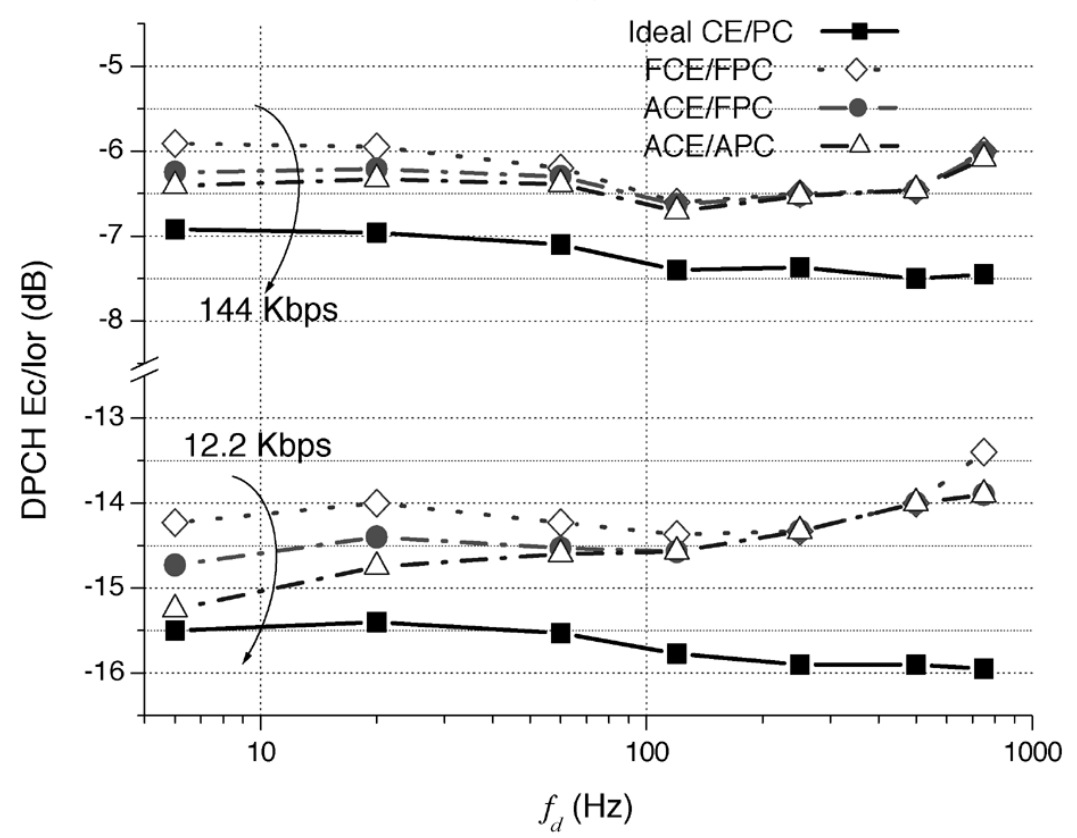

(b)

Fig. 6. Required DPCH $E_{c} / I_{o r}$ at a BER of $10^{-3}$ in the Case-3 channel with single transmit antenna. (a) $I_{o r} / I_{o c}=9 \mathrm{~dB}$ and $\beta_{c}^{2}=-13 \mathrm{~dB}$; (b) $I_{o r} / I_{o c}=$ $-3 \mathrm{~dB}$ and $\beta_{c}^{2}=-13 \mathrm{~dB}$.

It can also be seen that the DPCH gain is not much affected by the data transmission rate because the $\mathrm{CPICH}$ transmission rate is constant. The proposed ACE provides similar DPCH gain in the Case- 1 channel as in the Case- 3 channel. This implies that the performance gain of the proposed ACE is little affected by the number of multipaths.

It can be seen that the performance gain of the proposed SIR estimator over the FPC increases as $f_{d}$ decreases similar to that of the channel estimation. Note that the DPCH gain at $12.2 \mathrm{Kbps}$ is larger than that at $144 \mathrm{Kbps}$. This is because four dedicated pilot symbols are transmitted per slot for $12.2 \mathrm{Kbps}$ transmission, while eight pilot symbols are transmitted for 144 Kbps transmission. As the number of dedicated pilot symbols increases, the accuracy of one-slot averaged CIR estimate is improved. Thus, the advantage with the use of the adaptive scheme decreases. Since a significant amount of power redundancy is required for low-rate data transmission to allocate more than four pilot symbols per slot, the DPCH gain with the proposed SIR estimator increases as the data transmission rate decreases. Unlike with the use of the ACE, the DPCH gain with the proposed SIR estimator is little affected by $I_{o r} / I_{o c}$ since the SIR can be maintained by the DPCH transmit power control.

The implementation complexity is compared in Table II in terms of the required memory and multiplication operation. The amount of additional memory for the proposed scheme is negligible compared to the complexity of the rake receiver. 
TABLE II

IMPLEMENTATION COMPLEXITY

\begin{tabular}{|c|c|c|c|c|c|c|}
\hline $\mathrm{CE}$ & $\mathrm{CEF}$ & Type & Memory & Memory (example) & Multi./sec & Multi./sec (example) $\left(\times 10^{3}\right)$ \\
\hline \multirow{4}{*}{ Fixed CE } & \multirow{3}{*}{ CPICH CEF } & General FIR & $F M_{f}$ & 80 & $F M_{f} / \psi_{c} T_{c}$ & 1200 \\
\hline & & MA & $F M_{f}$ & 80 & $F / \psi_{c} T_{c}$ & 60 \\
\hline & & One-pole IIR & $F$ & 4 & $F / \psi_{c} T_{c}$ & 60 \\
\hline & DPCH CEF & 1-tap & & 4 & 0 & 0 \\
\hline \multirow{4}{*}{$\begin{array}{l}\text { Proposed } \\
\text { adaptive CE }\end{array}$} & CPICH CEF & MA & $F M_{\max }$ & 164 & $F / \psi_{c} T_{c}$ & 60 \\
\hline & & One-pole IIR & $F$ & 4 & $F / \psi_{c} T_{c}$ & 60 \\
\hline & DPCH CEF & MA & $F W_{\max }$ & 12 & $F / 2560 T_{c}$ & 6 \\
\hline & CPE & & $F\left(m_{\max }-M_{\max }\right)$ & 220 & $\frac{F\left(G_{l}+1\right)+\left(F G_{l}\right) / J}{\psi_{c} T_{c}}$ & 300.16 \\
\hline
\end{tabular}

* $M_{f}($ tap size of the fixed CPICH CEF $)=20, M_{\max }($ maximum tap size of the adaptive CPICH CEF $)=41, W_{\max }($ maximum tap size of the adaptive DPCH CEF $)=3, m_{\max }($ maximum correlator's interval $)=96, J$ (correlation period $)=1500(100 \mathrm{~ms}), F($ number of fingers $)=4$, and $T_{c}($ chip duration $)=2.6042 \times 10^{-7}$.

For example, the receiver should demodulate the transport format combination indicator including the information of the spreading factor and data bit size for proper demodulation of the data signal [2]. This requires one frame (ten slots) delay, necessitating a large number of memories (e.g., 6400 when 25600 chip symbols are stored after despreading with spreading factor of four) compared to that for channel estimation as seen in Table II. The proposed scheme requires the increase of the multiplication operation compared to the use of a fixed channel estimation scheme. However, it has computational complexity much less than that of other adaptive schemes with the use of CEF such as Wiener filter or general FIR filter. Thus, the proposed scheme can be realized without significant increase of implementation complexity.

\section{Channel Estimation AND SIR Measurement For OPEN-LOOP TWO-TRANSMIT ANTENNAS}

\section{A. Proposed Scheme}

With the use of ideal channel estimation, $\hat{s}_{1}^{\mathrm{DP}}[k]$ and $\hat{s}_{2}^{\mathrm{DP}}[k]$ can be represented as [23]

$$
\begin{gathered}
\hat{s}_{1}^{\mathrm{DP}}[k]=\beta_{c} \beta_{d} I_{o r} \sum_{l=0}^{L-1}\left\{\left(\left|h_{l, 1}[k]\right|^{2}+\left|h_{l, 2}[k]\right|^{2}\right) s_{1}[k]\right. \\
\left.+h_{l, 1}^{*}[k] z_{1, l}^{\mathrm{DP}}[k]+h_{l, 2}[k] z_{1, l}^{D P *}[k]\right\} \\
\hat{s}_{2}^{\mathrm{DP}}[k]=\beta_{c} \beta_{d} I_{o r} \sum_{l=0}^{L-1}\left\{\left(\left|h_{l, 1}[k]\right|^{2}+\left|h_{l, 2}[k]\right|^{2}\right) s_{2}[k]-h_{l, 2}[k]\right. \\
\left.\times z_{1, l}^{D P *}[k]+h_{l, 1}^{*}[k] z_{2, l}^{\mathrm{DP}}[k]\right\}
\end{gathered}
$$

Note that the antenna diversity effect can be obtained since the received signal consists of $2 L$ paths instead of $L$ paths. If the channel estimation is not perfect, $\hat{s}_{1}^{\mathrm{DP}}[k]$ and $\hat{s}_{2}^{\mathrm{DP}}[k]$ can be represented as [23]

$$
\begin{aligned}
\hat{s}_{1}^{\mathrm{DP}}[k]=\beta_{c} \beta_{d} I_{o r} & \\
\times \sum_{l=0}^{L-1}\{ & \left(\left|h_{l, 1}[k]\right|^{2} \varepsilon_{l, 1}^{*}[k]+\left|h_{l, 2}[k]\right|^{2} \varepsilon_{l, 2}[k]\right) s_{1}[k] \\
& +h_{l, 1}^{*}[k] z_{1, l}^{\mathrm{DP}}[k]+h_{l, 2}[k] z_{2, l}^{D P *}[k] \\
& +s_{2}^{*}[k]\left|h_{l, 1}[k]\right|\left|h_{l, 2}[k]\right| e^{-j \theta_{l, 1,2}[k]} \\
& \left.\times\left(\varepsilon_{l, 2}[k]-\varepsilon_{l, 1}^{*}[k]\right)\right\}
\end{aligned}
$$

$$
\begin{aligned}
\hat{s}_{2}^{\mathrm{DP}}[k]=\beta_{c} \beta_{d} I_{\text {or }} & \\
\times \sum_{l=0}^{L-1}\{ & \left\{\left(\left|h_{l, 1}[k]\right|^{2} \varepsilon_{l, 1}^{*}[k]+\left|h_{l, 2}[k]\right|^{2} \varepsilon_{l, 2}[k]\right) s_{2}[k]\right. \\
& -h_{l, 2}[k] z_{1, l}^{D P *}[k]+h_{l, 1}^{*}[k] z_{2, l}^{\mathrm{DP}}[k] \\
& +s_{1}^{*}[k]\left|h_{l, 1}[k]\right|\left|h_{l, 2}[k]\right| e^{-j \theta_{l, 1,2}[k]} \\
& \left.\times\left(\varepsilon_{l, 1}^{*}[k]-\varepsilon_{l, 2}[k]\right)\right\}
\end{aligned}
$$

where $h_{l, i}[k]=\left|h_{l, i}[k]\right| e^{-j \theta_{l, i}[k]}, \hat{h}_{l, i}[k]=\left|\hat{h}_{l, i}[k]\right| e^{-j \hat{\theta}_{l, i}[k]}$, $\hat{h}_{l, i}[k]=h_{l, i}[k] \varepsilon_{l, i}[k]$, for $i=1,2$, and $\theta_{l, 1,2}[k]=\theta_{l, 2}[k]-$ $\theta_{l, 1}[k]$. Thus, the channel estimate error can cause the crosstalk between $\hat{s}_{1}^{\mathrm{DP}}[k]$ and $\hat{s}_{2}^{\mathrm{DP}}[k]$. Moreover, the power of the $\mathrm{CPICH}$ pilot signal is split into two transmit antennas, resulting in less accurate channel estimate. Therefore, the receiver performance in the STTD scheme is much more affected by the channel estimation performance than in the single transmit antenna scheme.

The ACE proposed for single transmit antenna can be applied to the STTD scheme with a small modification. Since (18) requires two-symbol time interval, the tap size of the MA FIR CEF and the correlator's interval of the CPE need to be decreased by one-half, i.e., $m_{l, S T}=m_{l, N S T} / 2$ and $M_{l, S T}=M_{l, N S T} / 2$. By substituting $m_{l, S T}$ and $N_{l, S T}$ into (27), the optimum tap size of the MA FIR CEF in the STTD scheme can be determined by

$$
\hat{M}_{l, S T}=\left(\frac{8 \hat{m}_{l, S T}^{4}\left(v_{l}+\frac{I_{o c}}{I_{o r}}\right)}{\psi_{c} \beta_{c}^{2} S_{l} \hat{f}_{l}^{4}} \cdot \frac{1+K_{l}}{\frac{K_{l} \cos ^{4} \theta_{l}}{9}+\frac{1}{\chi_{l}}}\right)^{\frac{1}{5}} .
$$

Other procedures for the ACE design are the same as those in the single antenna. Similarly, the design of the SIR estimator for the STTD scheme can also be modified.

\section{B. Performance Evaluation}

Fig. 7 depicts the receiver performance in terms of the required DPCH $E_{c} / I_{o r}$ for a BER of $10^{-3}$ when the proposed scheme is employed under the same simulation condition as that of the single transmit antenna scheme. It can be seen that gain of the proposed ACE and SIR estimator over the FCE and FPC is much larger in the STTD scheme than in the single transmit antenna scheme. This is mainly due to the effect of split transmit power and crosstalk. 


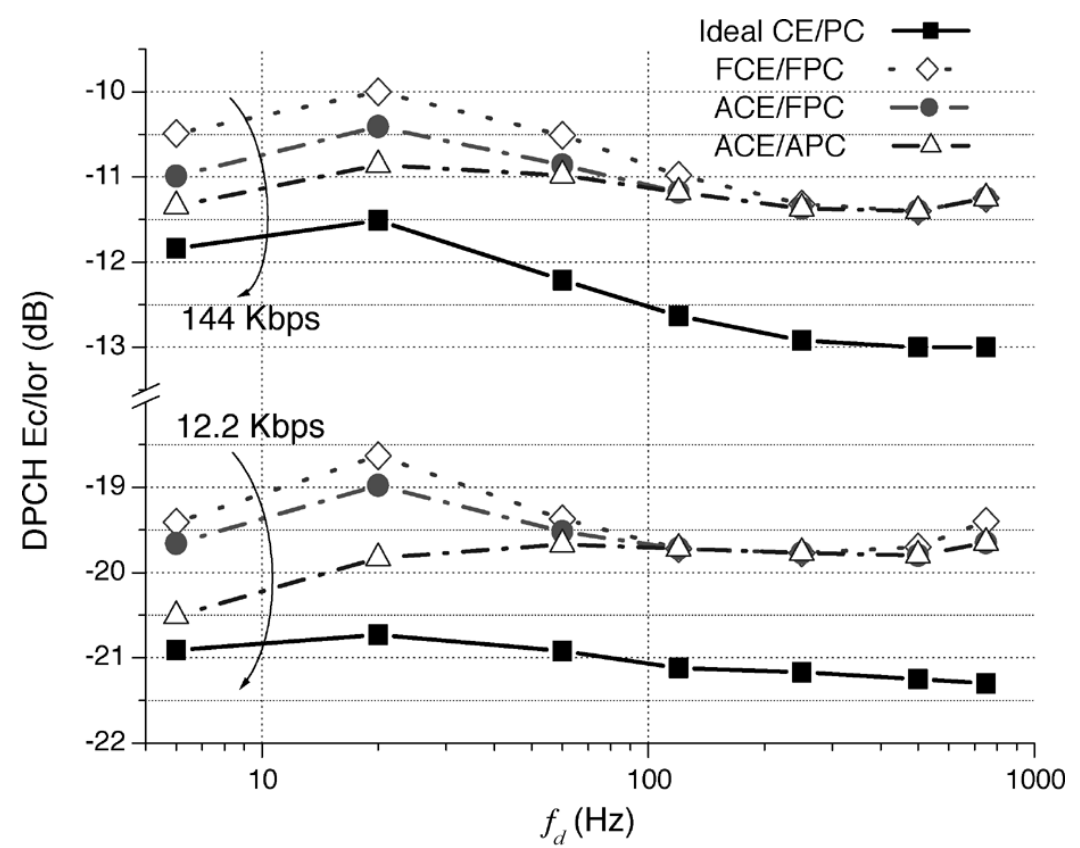

(a)

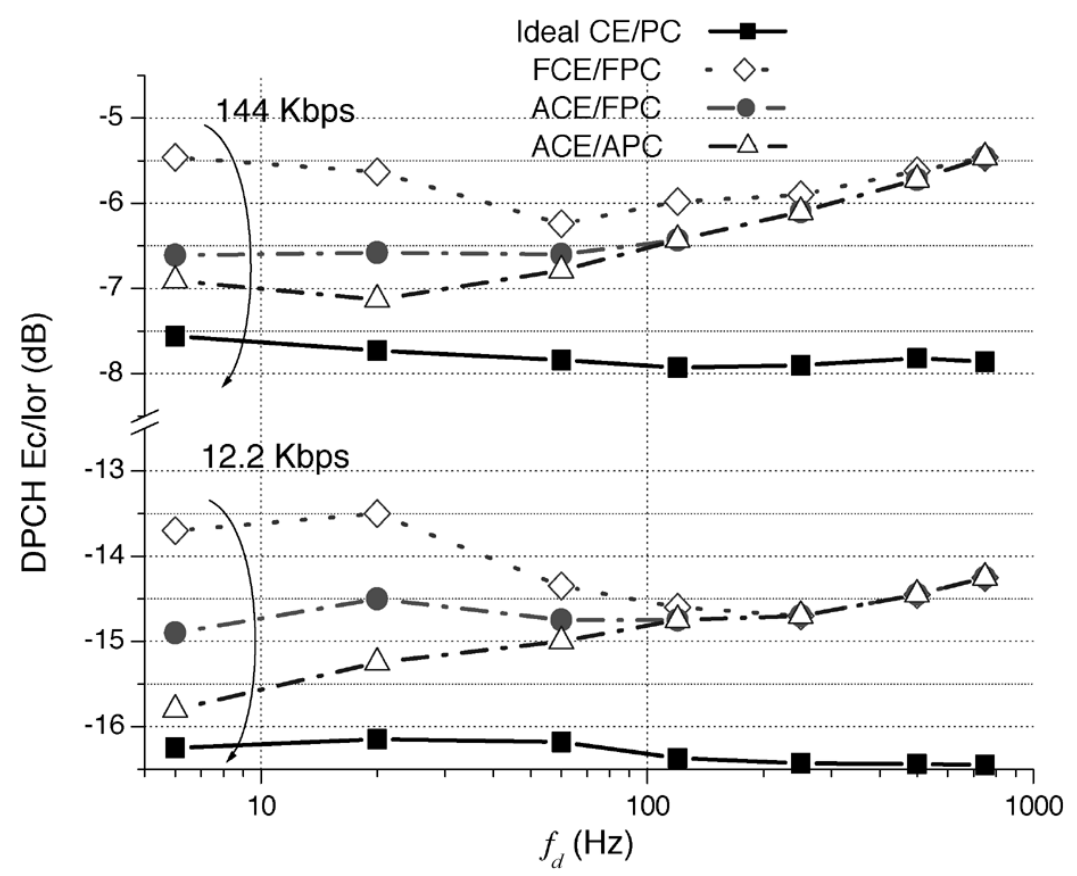

(b)

Fig. 7. Required DPCH $E_{c} / I_{o r}$ at a BER of $10^{-3}$ in the Case-3 channel with STTD. (a) $I_{o r} / I_{o c}=9 \mathrm{~dB}$ and $\beta_{c}^{2}=-13 \mathrm{~dB}$; (b) $I_{o r} / I_{o c}=-3 \mathrm{~dB}$ and $\beta_{c}^{2}=-13 \mathrm{~dB}$.

\section{CONCLUSION}

In this paper, we have proposed an adaptive channel estimator and a SIR estimator in the WCDMA downlink system that can work without exact a priori information on the operating condition. The proposed scheme adjusts the bandwidth (i.e., tap size) of the MA FIR CEF by exploiting the correlation characteristics of the received pilot signal. Numerical results show that the proposed scheme can provide significant performance improvement over the conventional fixed scheme for a wide range of channel variation, particularly under harsh channel condition with low $I_{o r} / I_{o c}$ or a small number of dedicated pilot symbols per slot. Due to the effect of split transmit power and crosstalk, further performance improvement can be achieved when a transmit antenna diversity scheme is employed. The proposed scheme can also be applied to other DS-CDMA systems that uses continuous common pilot signal and timemultiplexed user-dedicated pilot signal. 


\section{APPENDIX I}

DERIVATION OF (25)

By changing the summation form in (23) into an integral form, the MSE can be approximated by

$$
\begin{aligned}
\varepsilon_{l}\left(\kappa_{l}\right) \approx \beta_{c}^{2} I_{o r}\left(\frac{1}{\left(2 \kappa_{l}\right)^{2}} \int_{-\kappa_{l}}^{\kappa_{l}} \int_{-\kappa_{l}}^{\kappa_{l}} R_{h, l}(t-u) d t d u\right. \\
\left.-\frac{1}{\kappa_{l}} \int_{-\kappa_{l}}^{\kappa_{l}} R_{h, l}(t) d t+R_{h, l}(0)\right) \\
+\frac{\left(I_{o c}+v_{l} I_{o r}\right) T_{c p}}{2 \kappa_{l} \psi_{c}} \\
=\beta_{c}^{2} I_{o r}\left(\frac{1}{2 \kappa_{l}^{2}} \int_{0}^{2 \kappa_{l}} R_{h, l}(t)\left(\kappa_{l}-t\right) d t\right. \\
\left.-\frac{2}{\kappa_{l}} \int_{0}^{\kappa_{l}} R_{h, l}(t) d t+R_{h, l}(0)\right) \\
+\frac{\left(I_{o c}+v_{l} I_{o r}\right) T_{c p}}{2 \kappa_{l} \psi_{c}}
\end{aligned}
$$

where

$$
\kappa_{l}=\left(N_{l}+\frac{1}{2}\right) T
$$

and $R_{h, l}(\tau)$ is given by

$$
\begin{aligned}
R_{h, l}(\tau) & =E\left\{\operatorname{Re}\left(h_{l}^{*}(t) h_{l}(t+\tau)\right)\right\} \\
& =P_{l} \cos \left(2 \pi f_{d} \tau \cos \theta_{l}\right)+\sigma_{\alpha, l}^{2} R_{\alpha, l}(\tau) .
\end{aligned}
$$

Here, $R_{\alpha, l}(\tau)$ is the autocorrelaton of the scattered components in the $l$ th path signal given by [16]

$$
R_{\alpha, l}(\tau)= \begin{cases}J_{0}\left(2 \pi f_{d} \tau\right), & \text { for the classic spectrum } \\ \frac{\sin \left(2 \pi f_{d} \tau\right)}{2 \pi f_{d} \tau}, & \text { for the flat spectrum }\end{cases}
$$

where $J_{0}(\cdot)$ is the first kind, zeroth-order Bessel function.

The optimum $\kappa_{l}$ minimizing the MSE can be obtained by

$$
\begin{aligned}
\left.\frac{\partial \varepsilon_{l}\left(\kappa_{l}\right)}{\partial \kappa_{l}}\right|_{\kappa_{l}=\hat{\kappa}_{l}}=\beta_{c}^{2} I_{o r}\left(-\frac{1}{\hat{\kappa}_{l}^{2}} \int_{0}^{2 \hat{\kappa}_{l}} R_{h, l}(t) d t+\frac{1}{\hat{\kappa}_{l}^{3}} \int_{0}^{2 \hat{\kappa}_{l}} t R_{h, l}(t) d t\right. \\
\left.\quad+\frac{2}{\hat{\kappa}_{l}^{2}} \int_{0}^{\hat{\kappa}_{l}} R_{h, l}(t) d t-\frac{2}{\hat{\kappa}_{l}} R_{h, l}\left(\hat{\kappa}_{l}\right)\right) \\
-\frac{\left(I_{o c}+v_{l} I_{o r}\right) T_{c p}}{2 \hat{\kappa}_{l}^{2} \psi_{c}}=0 .
\end{aligned}
$$

Substituting a fourth-order Taylor approximation of cosine, sinc and Bessel functions into (39) for $0 \leq t \leq 2 \pi / 3, \hat{\kappa}_{l}$ can be obtained by

$$
\hat{\kappa}_{l}=\frac{1}{2}\left\{\frac{T_{c p}\left(I_{o c}+v_{l} I_{o r}\right)}{\left(\pi f_{d}\right)^{4} \psi_{c} \beta_{c}^{2} I_{o r}} \cdot \frac{1}{\frac{P_{l} \cos ^{4} \theta_{l}}{9}+\frac{\sigma_{\alpha, l}^{2}}{\chi_{l}}}\right\}^{\frac{1}{5}}
$$

where $\chi_{l}$ is a constant equal to 24 and 45 in the case of the classic and flat spectrum, respectively.
Denoting $S_{l}$ by the power ratio of the $l$ th path to total multipath signal, we have

$$
S_{l}=\frac{\left(P_{l}+\sigma_{\alpha, l}^{2}\right)}{\sum_{h=0}^{L-1}\left(P_{h}+\sigma_{\alpha, h}^{2}\right)} .
$$

Thus, $\hat{M}_{l}$ can be represented as

$$
\begin{aligned}
\hat{M}_{l}= & 2 \hat{\kappa}_{l} T_{c p} \\
= & \left(\frac{v_{l}+\frac{I_{o c}}{I_{o r}}}{\left(\pi f_{d} T_{c p}\right)^{4} \psi_{c} \beta_{c}^{2} S_{l}} \cdot \frac{P_{l}+\sigma_{\alpha, l}^{2}}{\sum_{h=0}^{L-1}\left(P_{h}+\sigma_{\alpha, h}^{2}\right)}\right. \\
& \left.\cdot \frac{1}{\frac{P_{l} \cos ^{4} \theta_{l}}{9}+\frac{\sigma_{\alpha, l}^{2}}{\chi_{l}}}\right)^{\frac{1}{5}} \\
= & \left(\frac{v_{l}+\frac{I_{o c}}{I_{o r}}}{\left(\pi f_{d} T_{c p}\right)^{4} \psi_{c} \beta_{c}^{2} S_{l}} \cdot \frac{1+K_{l}}{\frac{K_{l} \cos ^{4} \theta_{l}}{9}+\frac{1}{\chi_{l}}}\right)^{\frac{1}{5}} .
\end{aligned}
$$

\section{APPENDIX II}

\section{DERIVATION OF (27)}

Assuming that the prefiltered output $\bar{h}_{l}^{\mathrm{CP}}[n]$ is an egordic process and $m_{l} \geq N_{a}, w_{l}\left(m_{l}\right)$ can be approximated as

$$
\begin{aligned}
& w_{l}\left(m_{l}\right) \\
& \approx \frac{E\left\{\operatorname{Re}\left(\bar{h}_{l}^{C P *}[n] \bar{h}_{l}^{\mathrm{CP}}\left[n+m_{l}\right]\right)\right\}}{E\left\{\left|\bar{h}_{l}^{\mathrm{CP}}[n]\right|^{2}\right\}} \\
& =\frac{\beta_{c}^{2} I_{o r}\left\{P_{l} \cos \left(2 \pi f_{d} m_{l} T_{c p} \cos \theta_{l}\right)+\sigma_{\alpha, l}^{2} R_{\alpha, l}\left(m_{l} T_{c p}\right)\right\}}{\beta_{c}^{2} I_{o r}\left(P_{l}+\sigma_{\alpha, l}^{2}\right)+\frac{\left(I_{o c}+v_{l} I_{o r}\right)}{\left(N_{a} \psi_{c}\right)}} .
\end{aligned}
$$

Let $\hat{f}_{l}$ be the normalized maximum Doppler frequency

$$
\hat{f}_{l}=2 \pi f_{d} \hat{m}_{l} T_{c p}
$$

where $w_{l}\left(\hat{m}_{l}\right)=\eta$. Using a fourth-order Taylor approximation for the correlation function, it can be shown that

$$
a_{l} \hat{f}_{l}^{4}-b_{l} \hat{f}^{2}+c_{l}=0
$$

where

$$
\begin{aligned}
& a_{l}= \begin{cases}\frac{P_{l} \cos ^{4} \theta_{l}}{24}+\frac{\sigma_{\alpha, l}^{2}}{64}, & \text { Classic } \\
\frac{P_{l} \cos ^{4} \theta_{l}}{24}+\frac{\sigma_{\alpha, l}^{2}}{120}, & \text { Flat }\end{cases} \\
& b_{l}= \begin{cases}\frac{P_{l} \cos ^{2} \theta_{l}}{2}+\frac{\sigma_{\alpha, l}^{2}}{4}, & \text { Classic } \\
\frac{P_{l} \cos ^{2} \theta_{l}}{2}+\frac{\sigma_{\alpha, l}^{2}}{6}, & \text { Flat }\end{cases} \\
& c_{l}=(1-\eta)\left(P_{l}+\sigma_{\alpha, l}^{2}\right)-\eta \frac{\left(\frac{I_{o c}}{I_{o r}}+v_{l}\right)}{N_{a} \psi_{c} \beta_{c}^{2}} .
\end{aligned}
$$

Thus, $\hat{f}_{l}$ is determined by

$$
\hat{f}_{l}=\left(\frac{b_{l}-\sqrt{b_{l}^{2}-4 a_{l} c_{l}}}{2 a_{l}}\right)^{\frac{1}{2}} .
$$


Note that $\hat{f}_{l}$ is not a function of $f_{d}$ and depends upon other channel condition parameters. Using $\pi f_{d} T_{c p}=\hat{m}_{l}^{-1} \hat{f}_{l} / 2$, the optimum filter tap size $\hat{M}_{l}$ can be rewritten as a function of $\hat{m}_{l}$ as

$$
\hat{M}_{l}=\left(\frac{16 \hat{m}_{l}^{4}\left(v_{l}+\frac{I_{o c}}{I_{o r}}\right)}{\psi_{c} \beta_{c}^{2} S_{l} \hat{f}_{l}^{4}} \cdot \frac{1+K_{l}}{\frac{K_{l} \cos ^{4} \theta_{l}}{9}+\frac{1}{\chi_{l}}}\right)^{\frac{1}{5}} .
$$

\section{APPENDIX III \\ DECISION OF THE CPE PARAMETERS}

The lower bound of the filter tap size is obtained by substituting the parameters $S_{l, \max }, \chi_{l, \max }, K_{l, \min },\left|\theta_{l}\right|_{\max }, v_{l, \min }$, and $\left(I_{o r} / I_{o c}\right)_{\max }$ into (27). Similarly, the upper bound of the filter tap size is obtained by the value of $S_{l, \min }, \chi_{l, \min }, K_{l, \max }$, $\left|\theta_{l}\right|_{\min }, v_{l, \max }$, and $\left(I_{o r} / I_{o c}\right)_{\min }$, where the subscripts "max" and "min," respectively, denote the maximum and minimum values of the parameter.

The tap size of the first CEF $M_{l, 1}$ is set to a minimum tap size $M_{l, \min }$. Then, the value of $m_{l, 1}$ is determined from $M_{l, 1}$. Let us introduce lower bound margin factor $\lambda_{L}$ and upper bound margin factor $\lambda_{U}\left(0 \leq \lambda_{L}, \lambda_{U}<1\right)$ to have a freedom in the design of CPE. We determine $\lambda_{L}$ such that the point $A$ in Fig. 4 is represented as $\left(m_{l, 1}, M_{l, 1} /\left(1+\lambda_{L}\right)\right)$. It can be shown that

$$
\begin{aligned}
m_{l, i}=\left(\frac{M_{l, i}^{5} \psi_{c} \beta_{c}^{2} S_{l, \max } \hat{f}_{l, \max }^{4}}{16\left(1+\lambda_{L}\right)^{5}\left(v_{l, \min }+\left(\frac{I_{o c}}{I_{o r}}\right)_{\min }\right)}\right. & \left(\frac{\left(\frac{K_{l, \min } \cos ^{4}\left|\theta_{l}\right|_{\max }}{9}+\frac{1}{\chi_{l, \max }}\right)}{\left(1+K_{l, \min }\right)}\right)^{\frac{1}{4}}
\end{aligned}
$$

for $1 \leq i \leq G_{l}$. As $\lambda_{L}$ increases, the difference between the break points of the staircase shape (e.g., the point $C$ in Fig. 4) and the lower bound becomes larger. With $\lambda_{U}$ and $m_{l, 1}$, the tap size $M_{l, 2}$ of the second CEF in the $l$ th finger is determined such that $\left(m_{l, 1},\left(1+\lambda_{U}\right) M_{l, 2}\right)$ is on the upper bound (e.g., the point $B$ in Fig. 4). It can be shown that, for $1 \leq i \leq G_{l}$

$$
\begin{aligned}
M_{l, i+1}=\frac{1}{1+\lambda_{U}}\left(\frac{16 m_{l, i}^{4}\left(v_{l, \max }+\left(\frac{I_{o c}}{I_{o r}}\right)_{\max }\right)}{\psi_{c} \beta_{c}^{2} S_{l, \text { min }} \hat{f}_{l, \text { min }}^{4}}\right. \\
\left.\cdot \frac{\left(1+K_{l, \max }\right)}{\left(\frac{K_{l, \text { max }} \cos ^{4}\left|\theta_{l}\right|_{\min }}{9}+\frac{1}{\chi_{l, \text { min }}}\right)}\right)^{\frac{1}{5}} .
\end{aligned}
$$

Similarly, $m_{l, 2}$ can be calculated from $M_{l, 2}$ and (49) with $i=$ 2 . Thus, $M_{l, 3}$ can be calculated from $m_{l, 2}$ and (50) with $i=2$. In this way, $m_{l, i}, i=1,2, \ldots, G_{l}$ and $M_{l, i}, i=1,2, \ldots, G_{l}+$ 1 , can be determined in a sequential manner until the tap size becomes larger than a predetermined maximum value $M_{l, \max }$. When $M_{l, i+1} \geq M_{l, \max }, M_{l, i+1}$ is set to a value of $M_{l, \max }$.

In order to uniquely design the proposed ACE, we need to determine the values of $\eta, \lambda_{L}, \lambda_{U}, M_{l, \min }$, and $M_{l, \max }$. The threshold $\eta$ can be set to any positive value less than one since it has already been considered in the design of ACE by determining $c_{l}$ in (46). If the channel has a large number of mul- tipaths, the signal power is split into multiple paths. This effectively increases the denominator of (43), reducing the maximum value of the autocorrelation. Thus, it is desirable to set the threshold to a small positive value.

It is possible to increase the design freedom by adjusting the tap margin factor $\lambda_{L}$ and $\lambda_{U}$. The bandwidth of an MA FIR filter decreases as the tap size increases. If the filter tap size is smaller than the desired one, the output may suffer from excessive noise. On the other hand, when the tap size is much larger than the optimum one, the desired information can be filtered out, resulting in severe performance degradation. Therefore, it is desirable to determine the tap margins so that $0 \leq \lambda_{L}<\lambda_{U} \leq 1$ for conservative design. For example, we use $\lambda_{L}=0.0$ and $\lambda_{U}=0.5$ in Fig. 4.

The minimum tap size $M_{l, \text { min }}$ should be determined so that the receiver can operate even in the worst case (e.g., up to an allowable maximum Doppler frequency). In order to obtain near optimum performance in a very slow fading channel, the maximum tap size $M_{l, \max }$ should be determined so that the use of a CEF with a tap size larger than $M_{l, \max }$ can provide negligible performance improvement over the use of $M_{l, \max }$-tap MA FIR CEF.

\section{REFERENCES}

[1] T. Ojanpera and R. Prasad, Wideband CDMA for Third Generation Mobile Communications. Norwood, MA: Artech House, 1998.

[2] Physical Channels and Mapping of Transport Channels Onto Physical Channels (FDD), 3G TS 25.211, Jun. 2001.

[3] TIA, “The cdma2000 ITU-R RTT candidate submission,", Jun. 1998.

[4] F. Ling, "Coherent detection with reference symbol based channel estimation for direct sequence CDMA uplink communications," in Proc. VTC'93, May 1993, pp. 400-403.

[5] M. D. Srinath, P. K. Rajasekaran, and R. Viswanathan, Statistical Signal Processing With Applications. Englewood Cliffs, NJ: Prentice-Hall, 1996.

[6] V. Kaasila and A. Mammela, "Bit-error probability for an adaptive diversity receiver in a Rayleigh-fading channel," IEEE Trans. Commun., vol. 46, pp. 1106-1108, Sep. 1998.

[7] M. J. Barrett, "Error probability for optimal and suboptimal quadratic receivers in rapid Rayleigh-fading channels," IEEE J. Sel. Areas Commun., vol. COM-5, pp. 302-304, Feb. 1987.

[8] H. J. Oh and J. M. Cioffi, "An adaptive channel estimation scheme for DS-CDMA systems," in Proc. VTC'00 Fall, Sep. 2000, pp. 2839-2843.

[9] M. Sakamoto, J. Huoponen, and I. Niva, "Adaptive channel estimator with velocity estimator for W-CDMA receiver," in Proc. VTC'00 Spring, May 2000, pp. 2024-2028.

[10] G. Chen, X.-H. Yu, and J. Wang, "Adaptive channel estimation and dedicated pilot power adjustment based on the fading-rate measurement for a pilot-aided CDMA system," IEEE J. Sel. Areas Commun., vol. 19, pp. 132-140, Jan. 2001.

[11] M. Benthin and K. Kammeyer, "Influence of channel estimation on the performance of a coherent DS-CDMA system," IEEE Trans. Veh. Technol., vol. 46, pp. 262-268, May 1997.

[12] S. Abeta, M. Sawahashi, and F. Adachi, "Performance comparison between time-multiplexed pilot channel and parallel pilot channel for coherent rake combining in DS-CDMA mobile radio," IEICE Trans. Commun., vol. E81-B, no. 7, pp. 1417-1425, Jul. 1998.

[13] A. J. Viterbi, A. M. Viterbi, and E. Zehavi, "Performance of power-controlled wideband terrestrial digital communication," IEEE Trans. Commun., vol. 41, pp. 559-569, Apr. 1993.

[14] F. Kikuchi, H. Suda, and F. Adachi, "Effect of fast power control on forward link capacity of DS-CDMA cellular mobile radio," IEICE Trans. Commun., vol. E83-B, no. 1, pp. 47-55, Jan. 2000.

[15] Y.-S. Yoon and Y.-H. Lee, "Adaptive SIR estimation in WCDMA systems," in Proc. VTC'02 Spring, May 2002, pp. 275-279.

[16] W. C. Jakes, Microwave Mobile Communications. New York: Wiley, 1974.

[17] J.-W. Choi and Y.-H. Lee, "Adaptive channel estimation in DS-CDMA downlink systems," in Proc. PIMRC'02, Sept. 2002, pp. 1432-1436. 
[18] S. M. Alamouti, "A simple transmit diversity technique for wireless communications," IEEE J. Sel. Areas Commun., vol. 16, pp. 1451-1458, Oct. 1998.

[19] B. Yang, "Projection approximation subspace tracking," IEEE Trans. Signal Process., vol. 43, pp. 95-107, Jan. 1995.

[20] UE Radio Transmission and Reception (FDD), 3G TS 25.101, Jun. 2001.

[21] Joint Technical Committee, "RF channel characterization and system deployment modeling,", Tech. Rep., Sep. 1994.

[22] Guidelines for Evaluation of Radio Transmission Technologies for IMT2000, Rec. ITU-R M.1225, 1997.

[23] A. Correia, A. Hottinen, and R. Wichman, "Space-time transmitter diversity schemes for wideband CDMA," in Proc. VTC'OO Spring, May 2000, pp. 313-317.

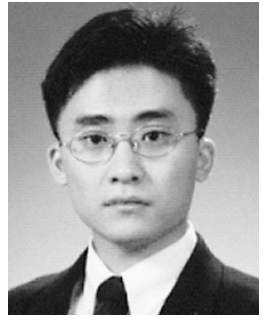

Ji-Woong Choi (S'00-M'04) received the B.S., M.S., and Ph.D. degree in electrical engineering from Seoul National University, Seoul, Korea, in 1998, 2000, and 2004, respectively.

Currently, he is with the Transceiver Technology Laboratory, Seoul National University, where he is studying the fourth-generation mobile system and pilot-assisted modulation. His research areas are wireless transmission systems including spread-spectrum and OFDM systems and signal processing for communication systems.

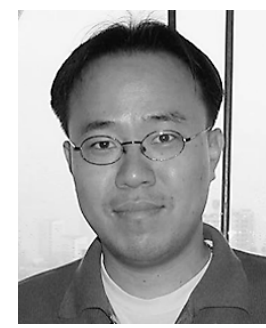

Young-Shin Yoon received the B.S. degree from Yonsei University, Korea, in 2000 and the M.S. degree from Seoul National University, Seoul, Korea, in 2002, all in electrical engineering.

Since 2002, he has been with SK Telecom, where he is currently a Researcher in the Terminal Development Team. His research interests include CDMA and SDMB transmission technique.

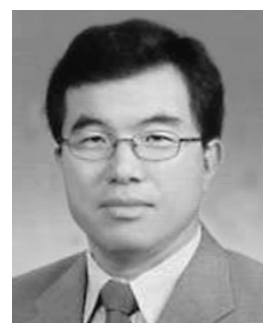

Yong-Hwan Lee (S'77-M'89) received the B.S. degree from Seoul National University (SNU), Korea, in 1977, the M.S. degree from Korea Advanced Institute of Science and Technology (KAIST) in 1980, and the Ph.D. degree from the University of Massachusetts, Amherst, in 1989, all in electrical engineering.

From 1980 to 1985 , he was with the Korea Agency for Defense Development as a Senior Engineer. From 1989 to 1994, he was with Motorola, Inc., as a Principal Engineer, where he was involved in the development of data transmission systems including high-speed modems. Since 1994, he has been with the School of Electrical Engineering, SNU, as a Faculty Member. He was Director of the Institute of New Media and Communications in SNU from 2001 to 2003 and is now a Vice Chair of the School of Electrical Engineering, SNU. He has been working on the design of wireline/wireless transceivers, including voiceband modems, power-line modems, wireless local loops, wide-band CDMA, and OFDM-based modems for next generation mobile communications. 